Abstract: In this paper, we report the experimental demonstration of an all-optical fiber-based $160-\mathrm{GHz}$ femtosecond pulse source exhibiting a duty cycle as low as $1 / 17$. The 380 -fs wellseparated Gaussian pulses are generated thanks to the strong temporal compression of an initial beat-signal propagating into three distinct segments of optical fiber. Experimental results are supported by numerical simulations based on the generalized nonlinear Schrödinger equation.

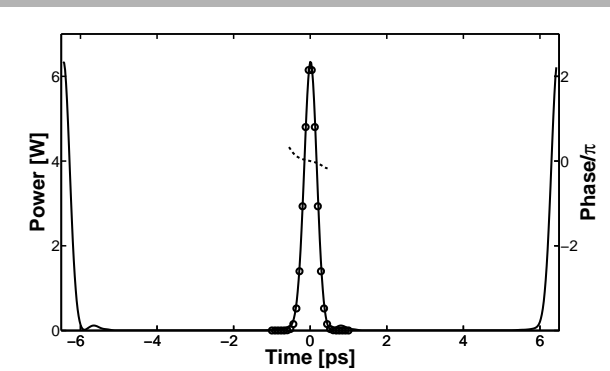

Intensity and phase profiles of the $160-\mathrm{GHz}$ output pulse train

\title{
All-Fibered High-Quality Low Duty-Cycle 160-GHz Femtosecond Pulse Source
}

\author{
Coraline Fortier, Bertrand Kibler, Julien Fatome *, Christophe Finot, Stephane Pitois and Guy Millot. \\ Institut Carnot de Bourgogne, UMR-CNRS 5209 Université of Bourgogne, 9 Avenue Alain Savary, 21078 Dijon, France
}

Received: 2008, Revised: 2008, Accepted: 2008

Key words: Nonlinear optics in fibers, femtosecond, low duty cycle, pulse compression

PACS: G1.10.Nz, 61.66.Bi

\section{Introduction}

Laser sources emitting optical pulses at repetition rates ranging from GigaHertz to TeraHertz around $1.5 \mu \mathrm{m}$ have stimulated a growing interest these last few years since they are involved in a host of applications such as optical sampling, component testing or ultra high capacity transmission system. A well-known and powerful technique used to generate such light sources relies on the temporal compression of a sinusoidal beating propagating in optical fiber line. Indeed, for more than ten years, this method has been successfully applied to generate femto- or picosecond pulse trains using various arrangements of optical fibers [1-15], such as modulational instability [1], dispersion decreasing fibers [2], step-like or comb-like fiber profiles [3-6]. Based on these previous results, Inoue et. al [3] have recently developed a 40 -steps pulse generation comblike setup that enables to transform an initial $160-\mathrm{GHz}$ beat-signal into a train of 320-fs well-separated pulses. In this work, we present an alternative approach to design a very low duty-cycle laser source emitting femtosecond pulses at a repetition rate of $160 \mathrm{GHz}$. The main advantage of our technique, previously demonstrated at 20- and
$40-\mathrm{GHz}[7,8]$, is that it allows to reduce the number of fiber sections to only three stages and reach a much higher output average power.

The first part of this paper is devoted to the presentation of the pulse source through numerical simulations based on the generalized nonlinear Schrödinger equation. Physical comprehension of the compression effect is obtained by showing evolution of the temporal and spectral pulse profiles all along the fiber line. Experimental results, showing the compression of the initial sinusoidal beating into a train of 380-fs pulses, are reported in the second part of the manuscript.

\section{Design and Numerical Simulations}

First, we study the nonlinear propagation of the initial 160$\mathrm{GHz}$ beat-signal generated by combining two linearly polarized continuous waves with different frequencies and equal powers. Our model is based on the generalized nonlinear Schrödinger equation, including fiber loss and higher-order effects such as third-order dispersion, self-

* Corresponding author: e-mail: julien.fatome@u-bourgogne.fr 


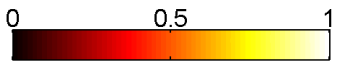

(a) Intensity [arb.unit.]

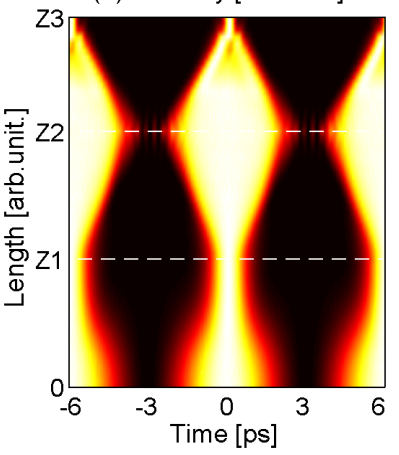

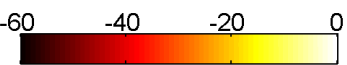

(b) Spectrum [dB]

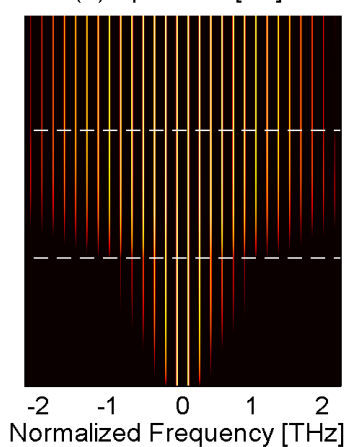

Figure 1 Numerical simulations results: (a) Temporal intensity evolution for two period of the pulse train, (b) Spectrum evolution in the three stages compressor.

steepening, and Raman scattering [9]. Our alternative approach for high-bit-rate pulse compression involves a simplified arrangement of commercially-available fiber segments with anomalous and normal dispersion [7]. The evolution of the beating is decomposed into three stages as illustrated in Figure 1. For each stage of the setup, some semi-empirical rules can be used to optimize and facilitate the design of our pulse train compressor [10]. The first stage of our setup aims to convert an initial sinusoidal beating into a well-separated Gaussian-like pulse train with a duty-cycle of $1 / 5$ through the multiple four-wave mixing process taking place into a standard nonzero dispersionshifted fiber (NZ-DSF) [10]. The second stage allows the passive reshaping of Gaussian pulses into a train of linearly chirped pulses thanks to the combined effects of self-phase modulation and normal dispersion. At the output of the second segment, the linear chirp of these pulses can therefore be compensated by simply adding an adequate length of anomalous dispersion fiber, which constitutes the final stage of the setup providing transformed-limited recompressed pulses.

Figure 1 and 3 shows the 160-GHz sinusoidal input signal evolving into compressed pulse train with $\sim 370$ fs FWHM pulses in both temporal and spectral domains. At the central wavelength of the initial beat signal, i.e., $\lambda_{0} \sim 1555.82 \mathrm{~nm}$, the NZ-DSF is assumed to have 0.21 $\mathrm{dB} / \mathrm{km}$ loss, an anomalous dispersion of $1 \mathrm{ps} / \mathrm{nm} / \mathrm{km}$, a nonlinear coefficient of $1.7 \mathrm{~W}^{-1} \cdot \mathrm{km}^{-1}$, and a third-order dispersion of $0.07 \mathrm{ps} / \mathrm{nm}^{2} / \mathrm{km}$. The optimum fiber length for achievement of maximum compression with minimum chirp and pedestal in the first compressor stage was found to be $\mathrm{Z} 1=2375 \mathrm{~m}$. We observe in Fig. 1 the temporal reshaping of the input sinusoidal signal accompanied by its spectral broadening through multiple four-wave mixing.

The choice of the second segment is a normal dispersion-flattened highly nonlinear fiber (NDF-HNLF) exhibiting losses of $1 \mathrm{~dB} / \mathrm{km}$, an anomalous dispersion of $-0.56 \mathrm{ps} / \mathrm{nm} / \mathrm{km}$, a nonlinear coefficient of $9 \mathrm{~W}^{-1} \cdot \mathrm{km}^{-1}$, and a third-order dispersion of $0.01 \mathrm{ps} / \mathrm{nm}^{2} / \mathrm{km}$. During the propagation in this segment, the pulses experience both temporal and spectral broadenings as well as a significant reshaping, with a flattening of the central part of the pulse [11]. A special attention was devoted to prevent temporal overlapping of consecutive pulses (due to the temporal broadening) which could lead to detrimental nonlinear interactions [12]. The optimum fiber length of the second stage is then $\mathrm{Z} 2=494 \mathrm{~m}$. The high nonlinearity of this type of fiber permits to achieve a strong spectral broadening as shown in Fig.1. Let us outline that the reduced third-order dispersion is crucial to maintain a symmetric broadening.

The final stage is based on a $17-\mathrm{m}$ long SMF-28 segment, assumed to have $0.22 \mathrm{~dB} / \mathrm{km}$ loss, an anomalous dispersion of $17 \mathrm{ps} / \mathrm{nm} / \mathrm{km}$, a nonlinear coefficient of $1.3 \mathrm{~W}^{-1} \cdot \mathrm{km}^{-1}$, and a third-order dispersion of 0.055 $\mathrm{ps} / \mathrm{nm}^{2} / \mathrm{km}$. As shown in Fig.1, the spectrum bandwidth remains constant, we then ensure a linear pulse recompression free from soliton effect compression [12]. All these parameters are those of the fibers used in our experiments.

The evolution of both intensity and chirp profiles for one period of the pulse train at different stages is shown in Fig.2(a,b). We confirm the conversion of the initial sinusoidal beating into Gaussian-like pulses with 1.22-ps FWHM duration (crosses), through the multiple four-wave mixing process. Fig.2(c) shows the corresponding optical spectrum fitted by a continuous Gaussian envelope with a FWHM bandwidth of $0.37 \mathrm{THz}$, giving a timebandwidth product of 0.45 , very close to the value of 0.441 corresponding to transformed-limited Gaussian pulses. Figure 2(a) confirms that the chirp across the pulses is nearly zero. Next, the second stage provides some linearly chirped pulses with $\sim 4.3$-ps FWHM duration (circles). In particular, we can note the emergence of some modulated pulse wings due to the beginning of the overlapping of consecutive pulses. This effect suggests that we have reached the optimum length of the second stage. Finally, the subsequent linear compression in the third stage leads to 370-fs Gaussian-like pulses (solid line) with an excellent symmetry. Although some residual energy below 5\% remains in low amplitude substructure in the wings, we here obtain lower pedestals than using soliton effect compression [9]. The combination of segments 2 and 3 has then enabled a 3.5 compression factor of the incoming Gaussian pulses. Fig.2(d) shows the Gaussian-like optical spectrum of the compressed pulses at the third stage output with $\sim 1.2-\mathrm{THz}$ FWHM bandwidth.

\section{Experimental Results}

A schematic of the experimental setup is shown in Figure 3. The initial beat-signal is generated by the superposition of two continuous waves delivered by two linearlypolarized external-cavity lasers (ECL) separated by 160 $\mathrm{GHz}$ around $1555 \mathrm{~nm}$ and combined by means of a 50:50 

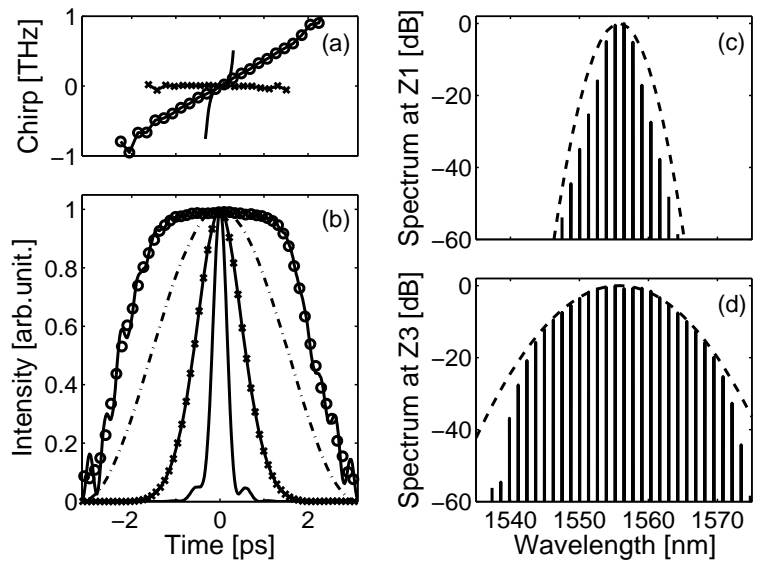

Figure 2 Numerical simulations results: Evolution of the chirp (a) and intensity (b) profiles at different stages of the setup (dashed line: compressor input, crosses: first stage output, circles: second stage output, solid line: compressor output). Optical spectra and their corresponding gaussian fit (dashed line) at both the first stage output (c) and the end of the pulse compressor (d).

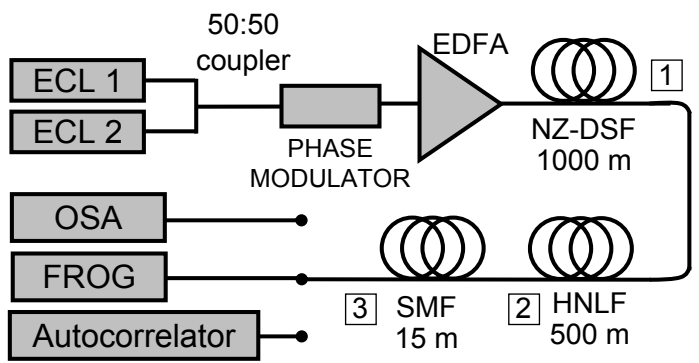

Figure 3 Experimental setup

coupler. A 100-MHz phase modulation is then applied to enlarge the diode linewidth so as to work far below the Brillouin scattering threshold. The beat-signal is finally amplified at an average power of $27 \mathrm{dBm}$ by means of a polarization-maintaining Erbium-doped fiber amplifier (EDFA) and injected into the first compression stage.

This first stage converts the initial beat-signal into a 1.3-ps Gaussian pulse train through a multiple four-wave mixing process taking place into a 1-km long NZ-DSF having an anomalous dispersion of $\mathrm{D}=1 \mathrm{ps} / \mathrm{km} . \mathrm{nm}$ [10]. The second stage, which involves $500 \mathrm{~m}$ of commerciallyavailable HNLF from OFS (parameters given in section 2), provides the passive reshaping of the Gaussian pulses into linearly chirped pulses, thanks to the combined effects of self-phase modulation and normal dispersion [13]. The final temporal compression is performed in the third stage : $17 \mathrm{~m}$ of SMF allows the compensation of the linear chirp and thus the generation of ultrashort quasi-gaussian pulses. At the output of the fiber line, the resulting pulses were analyzed by means of an SHG-based autocorrelator, an opti-
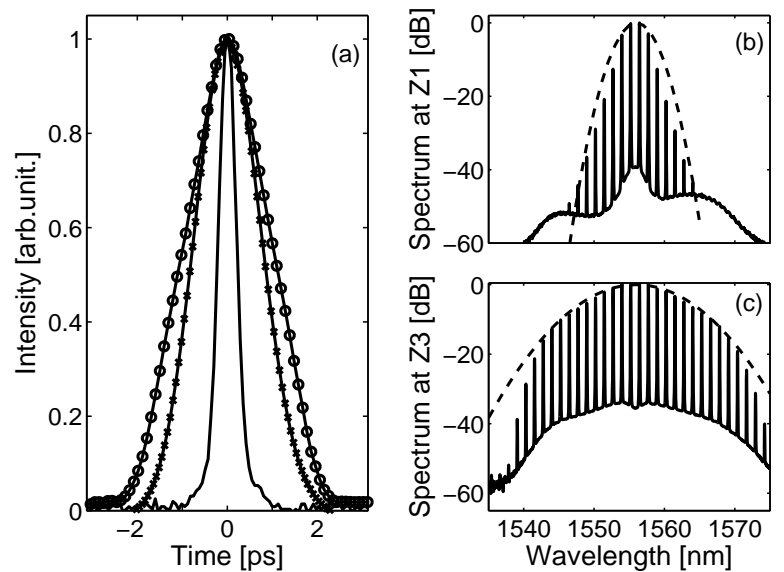

Figure 4 (a) : Evolution of autocorrelation function at different stages of the fiber line, crosses: first stage output, circles : second stage output and solid line :compressor output. (b) solid line : optical spectrum at the first stage output, dashed line : Gaussian fit. (c) solid line : optical spectrum at HNLF output, dashed line : Gaussian fit.

cal spectrum analyzer (OSA) and a home-made frequencyresolved optical gating (FROG) setup [15]. We can notice $\pi$-jump phase between two consecutive pulses which originates from the initial beat-signal.

We have represented in 4 the experimental autocorrelation results at each step of the fiber compressor line. Crosses in Fig. 4a represents the 1.3-ps Gaussian pulses generated at the first fiber output thanks to the nonlinear compression of the initial sinusoidal beating. The corresponding spectrum, visible in Fig. 4b, reveals the large number of sidebands generated by the four-wave mixing process which takes place in this NZ-DSF [14].

The spectrum at the output of the HNLF (second stage) is shown in Fig. 4c. As can be seen circles in Fig. 4a, the transformation of the Gaussian pulses into linearly chirped pulses in the normally dispersive fiber is accompanied by a large broadening of the intensity spectrum.

Finally, solid line in Fig. 4a represents the autocorrelation profile at the fiber line output, after the quasilinear temporal compression which occurs in the last piece of fiber (SMF). Assuming Fourier transformed-limited pulses, one may infer a FWHM temporal width equal to 380 -fs. We have also observed that the spectra after the second- and third-stage outputs are nearly the same. This result is not surprising since light propagation in the last stage is essentially dominated by dispersive (linear) effects. Moreover, we have found that this output spectrum can be accurately fitted by a Gaussian enveloppe (dashed line in Fig. 4c) having a full-width at half-maximum of 1.1 THz. The Time-Bandwidth product of 0.45 is fully consistent with the value 0.44 usually associated with Fourier limit Gaussian pulses. 


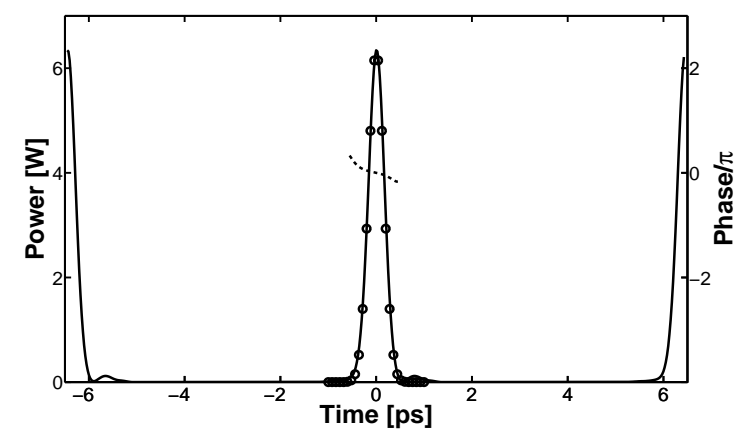

Figure 5 a) Intensity and phase profile of $160-\mathrm{GHz}$ pulse train at stage 3 obtained by FROG measurement.

This last result was confirmed by the FROG measurements represented in Fig. 5. The retrieved intensity profile (solid line) confirms that the $160-\mathrm{GHz}$ pulses have a Gaussian shape with a FWHM of $380 \mathrm{fs}$. Given the period of $6.25 \mathrm{ps}$, this constitutes a very low duty cycle of $1 / 17$. The retrieved phase (dashed line) is also pretty flat, confirming that pulses are nearly transform-limited. Finally, the available peak power exceeds $6 \mathrm{~W}$, which is quite high compared to ref. [3], essentially because of a large reduction of the number of fiber sections spliced together. This then leads to an output average power as high as $400 \mathrm{~mW}$ which is beneficial in the context of optical nonlinear processing. We obtained high peak-to-pedestal ratio of more than $18 \mathrm{~dB}$.

\section{Conclusion}

In conclusion, we have generated and characterized a 160$\mathrm{GHz}$ Gaussian pulse source having a FWHM of $380 \mathrm{fs}$ corresponding to a very low duty-cycle of $1 / 17$. The experimental setup is based on the temporal compression of an initial beat-signal into only three stages of optical fiber, validating this alternative method to comb-like dispersion decreasing techniques. We finally believe that this kind of sources could find many applications in the field of ultrafast optics such as optical sampling or ultrahigh capacity transmission.

\section{Acknowledgment}

We would like to acknowledge financial support of the Agence National de la Recherche (Projet ILIADE).
[2] S.V.Chernikov, E.M.Dianov, D.J.Richardson, R.I.Laming, and D.N.Payne, Appl. Phys. Lett. 63, 293-295 (1993).

[3] T.Inoue, and S.Namiki, Laser Photon. Rev. 2, 83-99 (2008).

[4] S.V.Chernikov, J.R.Taylor, and R.Kashyap, Opt. Lett. 19 539-541 (1994).

[5] Y.Ozeki, S.Takasaka, T.Inoue, K.Igarasho, J.Hiroishi, R.Sugizaki, M.Sakano, and S.Namiki, IEEE Photon. Technol.Lett.17, 1698-1700(2005).

[6] S.V.Chernikov, J.R.Taylor, and R.Kashyap, Electron. Lett. 30, 433-435 (1994).

[7] C.Finot, J.Fatome, S.Pitois, and G.Millot, Phot. Tech. Lett. 19, 1711-1713 (2007).

[8] S. Pitois, C. Finot, J. Fatome, B. Sinardet, and G. Millot,Opt. Commun. 260, 301-306 (2006).

[9] G.P. Agrawal, Nonlinear Fiber Optics, Academic Press, San Francisco (2001);

[10] S.Pitois, J.Fatome, and G.Millot, Opt. Lett. 27, 1727-1731 (2002).

[11] C. Finot, B. Kibler, L. Provost and S. Wabnitz, submitted to J. Opt. Soc. Am. B.;

[12] J.E. Rothenberg, Opt. Lett. 15, 443-445 (1990).

[13] W.J.Tomlinson, R.H.Stolen, and C.V.Shank, J. Opt. Soc. Am. B 1, 139-149 (1984).

[14] J.Fatome, S.Pitois, and G.Millot, IEEE J. Quant. Elect. 42, 1038-1046 (2006).

[15] J. Fatome, S. Pitois, and G. Millot, Opt. Fiber. Technol. 10, 73-78 (2004);

\section{References}

[1] A.Hasegawa,Opt. Lett., 9, 288-290 (1984). 